\title{
The use of a combination frequency technique to measure the surf zone bubble population
}

\author{
Andy D. Phelps, ${ }^{\text {a) }}$ David G. Ramble, and Timothy G. Leighton \\ Institute of Sound and Vibration Research, University of Southampton, Southampton SO17 1BJ, \\ United Kingdom
}

(Received 1 July 1996; accepted for publication 16 October 1996)

\begin{abstract}
There are great benefits to sizing bubbles using a two frequency technique, which examines the appearance of sum-and-difference signals generated by the interaction between a resonant bubble pulsation and a much higher frequency imaging beam. This paper presents the results from using the technique to size bubbles in the ocean surf zone, and details the pulsation model used to calibrate the returned data such that the height of the bubble scattered signal can be related to the number of resonant bubbles of that size. It also shows how ambiguities and inaccuracies (brought on through turbulence and the substantial off-resonance nature of the signal) which affected earlier oceanic tests using the same method can be identified in the returned signal or removed from the estimate during the data processing. (C) 1997 Acoustical Society of America. [S0001-4966(97)00604-8]
\end{abstract}

PACS numbers: 43.30.Pc, 43.30.Lz, 43.25.Yw [JHM]

\section{INTRODUCTION}

There are many practical applications where a knowledge of the size and distribution of a bubble population would be of benefit, ${ }^{1-3}$ and the strong backscattering properties due to the impedance mismatch at the bubble surface makes acoustic measurements particularly amenable to the task. When excited by sound, bubbles pulsate volumetrically as a single degree of freedom system, where the inertia arises from fluid around the bubble, the stiffness through gas compression inside the bubble, and the damping is brought on through viscous losses at the wall, thermal dissipation into the fluid, and acoustic radiation. These volumetric pulsations have a well-defined acoustic resonance frequency, given by ${ }^{4}$

$$
\omega_{0} \approx \frac{1}{R_{0} \sqrt{\rho}} \sqrt{3 \kappa\left(p_{0}+\frac{2 \sigma}{R_{0}}\right)-\frac{2 \sigma}{R_{0}}-\frac{4 \mu^{2}}{\rho R_{0}^{2}}},
$$

where $\omega_{0}$ is the resonant frequency in $\mathrm{rad} / \mathrm{s}, R_{0}$ is the equilibrium bubble radius, $\rho$ is the density of the surrounding fluid (assumed to be incompressible), $\kappa$ is the polytropic index of the gas compression, $p_{0}$ is the hydrostatic pressure at the bubble wall, $\sigma$ is the surface tension of the gas/liquid interface, and $\mu$ is the shear viscosity coefficient of the liquid.

It is possible to measure the resonance frequencies of a bubble population through observing the strength of a backscattered acoustic signal, ${ }^{5}$ which is assumed to be a maximum when the driving signal frequency, here called the "pump" frequency $\omega_{p}$, is coincident with a bubble resonance. However, such estimates have poor spatial resolution, since at resonance the radii of bubbles are orders of magnitude smaller than the wavelength of the sound field, and provide ambiguous results, in that a bubble much larger than resonance may scatter more sound than a smaller resonant bubble. ${ }^{4}$ This ambiguity may by reduced by monitoring the nonlinear behavior of a bubble, because at large pulsation

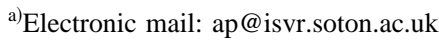

amplitude (typically taken to be an indicator of resonance) the bubble motion becomes increasingly asymmetric: As an obvious example, a bubble can theoretically expand without limit but only contract to zero radius. This results in integer related harmonics of the driving signal frequency at $2 \omega_{p}$, $3 \omega_{p}$, etc. It has been demonstrated, ${ }^{6}$ however, that exploitation of these particular nonlinear effects suffers in that the signals are difficult to distinguish from other harmonic distortion in the measuring equipment, and also fail to address the problem of poor spatial resolution. Other noninteger harmonics of the sound field, typically a subharmonic at $\omega_{p} / 2$ and ultraharmonics at $3 \omega_{p} / 2,5 \omega_{p} / 2$, etc., are also stimulated near resonance. These have been shown to be unambiguous indicators of a resonant bubble and provide greater accuracy in determining its resonance frequency owing to the much reduced off-resonance signal contribution. ${ }^{7}$ However, these signals are impractical for a generalized bubble sizer when stimulated at resonance as they are parametric in nature, and arise from surface effects which do not propagate into the medium. Specifically, the subharmonic at $\omega_{p} / 2$ arises through Faraday waves set up on the bubble surface, ${ }^{7}$ which do not bring about a volumetric change in the bubble and therefore do not emit as monopoles.

Many of these limitations have been overcome through use of a two frequency technique, where a bubble population is simultaneously insonified with a high-frequency fixed signal $\omega_{i}$ (the "imaging" signal) as well as the lower frequency signal $\omega_{p}$ intended to drive the population at their resonant frequencies. ${ }^{8}$ When this low-frequency sound field is coincident with a bubble resonance and the wall pulsations are large, the high-frequency sound scattered from the bubble is amplitude modulated at this frequency, and this gives rise to sum-and-difference components in the returned signal at $\omega_{i} \pm \omega_{p}$. Therefore the technique allows the exploitation of the basic resonant behavior of a bubble, but without the potential ambiguity caused when a large bubble is mistaken for a small resonant bubble. Additionally, the use of combination frequency measurements allows very specific spatial lo- 
calization, and the signal-to-noise ratio is greatly improved as the process translates only bubble-mediated information from the noisy frequency window around their resonance up to the comparatively quieter frequency window around the imaging signal.

This particular method of sizing bubbles has been investigated by earlier workers for laboratory populations employing increasingly more sophisticated signal processing techniques, ${ }^{8-10}$ and once on an oceanic population. ${ }^{11}$ The oceanic data was taken using a chirped signal between 2.5 and $6 \mathrm{kHz}$, with an imaging frequency of $450 \mathrm{kHz}$, but in the tests no distinction was made between bubble-mediated coupling and that caused by turbulence, or compensation for the significant off-resonance contribution which is characteristic of the $\omega_{i} \pm \omega_{p}$ signal, and the pump transducer frequency response. This paper describes the results of work undertaken to build a more robust and accurate bubble population estimator, and describes results collected in oceanic surf zone measurements, taken at four spot frequencies of 28, 50, 60 , and $88 \mathrm{kHz}$. The choice of three of these frequencies allows comparison of the returned data with earlier oceanic bubble population estimates taken exploiting the resonant backscatter effect. ${ }^{5}$ The paper shows how turbulent effects are differentiated from the bubble signals, and demonstrates how the off-resonant contribution to the $\omega_{i} \pm \omega_{p}$ signal can find compensation in the calibration of the equipment. As individual tones were employed, the variable frequency response of the source transducer could be removed, and constant and clearly defined bubble insonification conditions could be chosen. The paper first describes the theoretical model used in the estimation of the strength of the signal coupling, then proceeds to describe the calibration of the equipment. The experimental oceanic setup is discussed, with the results collected on site presented, analyzed, and compared with historical data.

\section{BUBBLE RESPONSE TO TWO FREQUENCY INSONIFICATION}

There are a number of different bubble models currently available which can be used to calculate radius-time curves for pulsating bubbles, and which differ from each other in complexity and in the range of insonification amplitudes and bubble size over which they are applicable. One of the best known and simplest of these models is the Rayleigh-Plesset equation, which considers the volumetric pulsations of an assumed spherical bubble existing in a infinite and incompressible medium. ${ }^{12}$ Although this model is nonlinear and can only be solved numerically, there are certain simplifying assumptions which can be made to allow an analytical solution. This was the subject of the analysis performed by Zabolotskaya and Soluyan ${ }^{13}$ and Newhouse and Shankar, ${ }^{8}$ who derived expressions for the pressure amplitudes at the various frequency locations through consideration of a small volumetric or radial perturbation. However, their analyses did not account fully for the various damping mechanisms that affect the radial pulsations of a bubble, and which are of considerable importance when estimating its resonant behavior.
There are three damping mechanisms which have been identified and theoretically considered, and they must all be included in any full consideration of the resonant properties of a pulsating bubble. These are losses through viscous action at the bubble wall, thermal conduction between gas and liquid, and energy dissipation through acoustic radiation. Of these, only viscous losses were included in the original theory: ${ }^{8}$ the gas was considered to behave adiabatically and no account was taken of the energy radiation into the fluid brought about through the passage of the sound through a compressible medium. However, over the range of bubble sizes which were examined in the earlier work, ${ }^{8}$ and are examined in this paper, viscous losses are orders of magnitude smaller than the other two damping mechanisms, and thus using the expression to calculate the expected amplitudes of the various signals at resonance will yield erroneous results. It should be noted that Newhouse and Shankar ${ }^{8}$ identified this potential for error, and in comparing measured results to their theory they left the total damping coefficient $\delta$ as a variable which was then altered to provide a least squares fit to their data.

For the theory applied in calibrating the oceanic sizer, the work of Newhouse and Shankar is extended to include explicitly for these extra damping mechanisms. A corrected form of the Rayleigh-Plesset equation is used as a starting expression, which includes an extra term which has been shown to give a reliable measure of the dissipative effects of sound radiation through a compressible medium. ${ }^{14}$ Additionally, the thermal conduction into the fluid is included in an approximate manner by assuming that the pulsations behave polytropically, with an index $\kappa$ calculated theoretically using the expressions of Eller. ${ }^{15}$ The modified form of the Rayleigh-Plesset differential equation is

$$
\rho R \ddot{R}+\frac{3}{2} \rho \dot{R}^{2}=p_{B}(t)-p_{0}-P(t)+\frac{R}{c}\left(1-\frac{\dot{R}}{c}\right) \frac{d p_{B}}{d t},
$$

where $R$ is the instantaneous bubble radius, $c$ is the speed of sound in the liquid, and $P(t)$ is the acoustic driving term. The remaining term $p_{B}(t)$ is a measure of the pressure immediately outside the bubble wall, and represents the forcing term on the liquid due to the bubble which the acoustic pressure has to overcome. It is given by

$$
p_{B}(t)=\left(p_{0}+\frac{2 \sigma}{R_{0}}\right)\left(\frac{R_{0}}{R}\right)^{3 \kappa}-\frac{2 \sigma}{R}-\frac{4 \mu \dot{R}}{R} .
$$

Following the earlier methodology, an approximate solution to this expression is sought by considering a small radial perturbation, where the variable $R$ can be rewritten in terms of a displacement variable $x$ as

$$
R=R_{0}(1+x) \quad \text { with } x \ll 1 \text {. }
$$

Using this substitution, and neglecting all terms beyond those in $x^{2}$, Eq. (2) can be rewritten as: 


$$
\begin{aligned}
\rho R_{0}^{2}(1+x) \ddot{x}+\frac{3}{2} \rho R_{0}^{2} \dot{x}^{2}= & \left(p_{0}+\frac{2 \sigma}{R_{0}}\right)\left[1-3 \kappa x+\frac{3}{2} \kappa(3 \kappa+1) x^{2}\right]-\left[p_{0}+\left(\frac{2 \sigma}{R_{0}}\right)\left(1+x+x^{2}\right)\right] \\
& -4 \mu(\dot{x}-x \dot{x})+p_{1} \cos \omega_{1} t+p_{2} \cos \omega_{2} t+\frac{R_{0}}{c}\left\{3 \kappa \dot{x}\left(p_{0}+\frac{2 \sigma}{R_{0}}\right)\left(3 \kappa x+\frac{R_{0}}{c} \dot{x}-1\right)\right. \\
& \left.+4 \mu\left(\dot{x}^{2}-\ddot{x}+\frac{R_{0}}{c} \dot{x} \ddot{x}\right)+2 \sigma \dot{x}\left(\frac{1}{R_{0}}-\frac{x}{R_{0}}-\frac{\dot{x}}{c}\right)\right\}
\end{aligned}
$$

where $\omega_{1}$ and $\omega_{2}$ are the two angular frequencies of the sinusoidal driving sound fields and $p_{1}$ and $p_{2}$ are their corresponding pressure amplitudes. If the acoustic radiation terms (shown grouped in curly brackets) are removed by considering the incompressible case of $c \rightarrow \infty$, this equation becomes Eq. (5) from Newhouse and Shankar's paper. ${ }^{8}$ The subsequent analysis follows the method in their paper of writing a solution to the displacement variable $x$ in terms of the sum of radial excursions at $\omega_{1}, \omega_{2}, 2 \omega_{1}, 2 \omega_{2}, \omega_{1}+\omega_{2}$, and $\omega_{1}-\omega_{2}$, substituting this into the modified Rayleigh-Plesset equation, and then equating the terms at the various different frequencies. The analytical solution is readily obtained (but cannot be simplified to obtain a transparent expression of the form obtained by Newhouse and Shankar, ${ }^{8}$ and is therefore not presented here), and its use is described in the next section. It is the case, however, that the results for the radiated pressures at the sum-and-difference frequency locations are numerically equal to those obtained by augmenting the damping term given in Newhouse and Shankar's Eq. (8) with the expression for the nondimensional radiated damping coefficient presented by Eller. ${ }^{15}$ If the more general offresonance form for the viscous damping is employed, then their variable $\delta$ can be replaced with the general damping parameter $d_{\text {tot }}$ given by

$$
d_{\mathrm{tot}}=\frac{4 \omega_{2} \mu}{3 \kappa p_{0}}+\frac{\rho}{3 \kappa p_{0}} \frac{\left(R_{0} \omega_{2}\right)^{3}}{c}
$$

where $\omega_{2}$ is considered to be the angular frequency of the pump sound field.

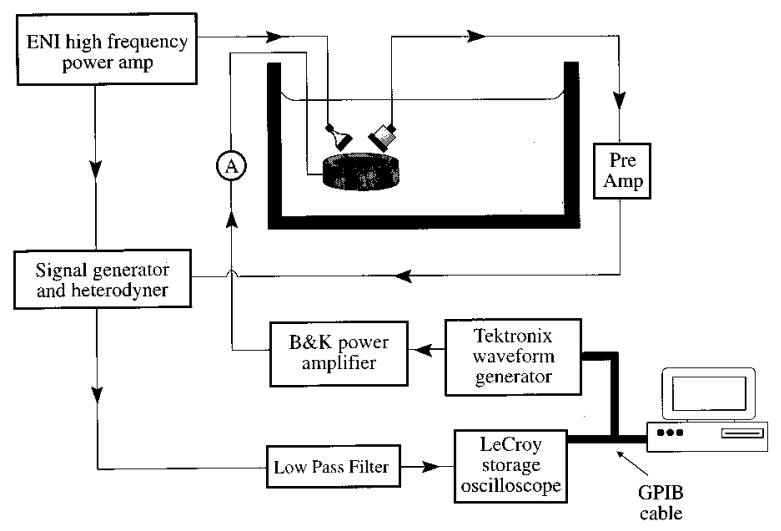

FIG. 1. Schematic of the equipment used in the laboratory tests.

\section{EQUIPMENT AND APPARATUS}

\section{A. Laboratory calibration}

The calibration of the apparatus was performed both to test that the pulsation model described above presented correct predictions for the relative heights of the different frequencies in the returned signal, and to enable the absolute height of the signals in the oceanic tests to be related to the signal strength associated with one resonant bubble. This was achieved by repeatedly insonifying a steady bubble stream of known resonant frequency, and examining the form of the returned signal. The laboratory tests were performed in a $1.8-\mathrm{m} \times 1.2-\mathrm{m} \times 1.2$-m-deep glass reinforced plastic tank which is vibration isolated from the floor, and is filled with tap water to $1-\mathrm{m}$ depth. The equipment schematic is shown in Fig. 1, and a detail of the transducer arrangement is included in Fig. 2. This is the same transducer head which was later used in the oceanic tests, and it will be demonstrated that fixing the geometry in this way allows certain parameters in the pulsation model to be poorly defined without any loss of accuracy.

The pump frequency signal generation was achieved using a Tektronix 2005 arbitrary waveform generator controlled via a GPIB cable connected to a PC, which was passed into a Bruel \& Kjaer 2713A power amplifier. The pump transducer comprised a 104-mm-i.d. piezoceramic ring transducer which was set into a polyurethane foam and encased in a nylon housing. The imaging signal was generated by a $1-\mathrm{MHz}$ crystal oscillator amplified with an ENI 240L rf power amplifier, and this was passed to the imaging signal transducer-the head of a Therasonic 1030 ultrasonic therapy unit as manufactured by Electro Medical Supplies,

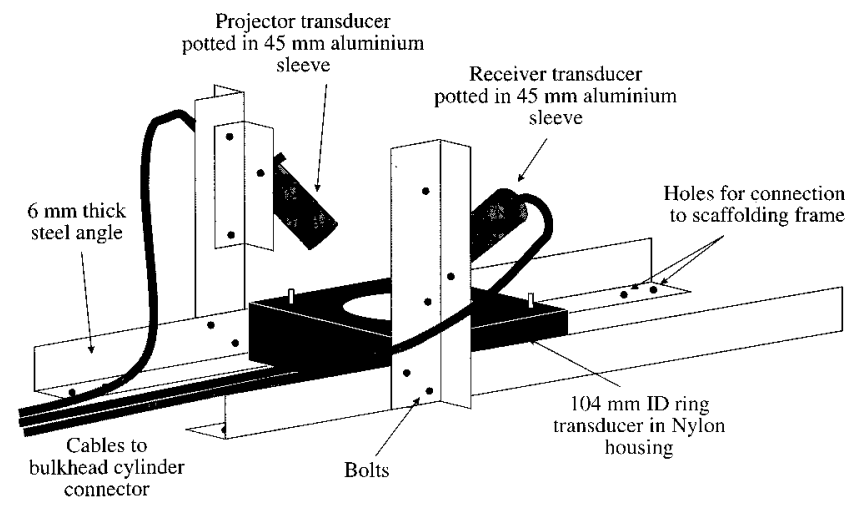

FIG. 2. Close up of the transducer arrangement used in both the laboratory tests and the oceanic measurements. 
and which was potted inside a 45-mm-diam aluminum cylinder to protect it when used in the open sea. The imaging signal amplitude at the focus of the two transducers was measured as $30 \mathrm{kPa}$ using a calibrated needle hydrophone (active element diameter $=0.5 \mathrm{~mm}$ ) with a HP1 submersible preamplifier, as manufactured by Precision Acoustics Ltd.

The returned signal from the bubble was monitored using a Panametrics V302 piezoceramic transducer, similarly potted in a 45-mm-diam sleeve, and conditioned using a Panametrics 5670 preamplifier. The preamplified signal was then heterodyned with a dummy signal from the crystal oscillator: this results in the useful information contained just above and below the imaging frequency being reproduced at just above dc, enabling much lower sampling rates and data storage. The low-frequency information was filtered to prevent aliasing using a Barr and Stroud EF5-02 $46 \mathrm{~dB}$ /octave filter and acquired on a LeCroy 9314L storage oscilloscope. For the laboratory tests the data were sampled at $50 \mathrm{kHz}$ and 10000 points taken. The beam patterns of the two highfrequency transducers were modeled by performing a Rayleigh integral over their surfaces. When these patterns were overlapped in a similar layout to the transducer arrangement, they allowed the insonification volume to be estimated. This gave an insonification volume, defined by where the sensitivity fell off to $3 \mathrm{~dB}$ of its peak value, of $0.2 \mathrm{~cm}^{3}$, demonstrating the potential of the technique to achieve high spatial resolution. Because of this, it should be noted that the equipment cannot provide depth profiles of the bubble population, although it is conceivable that a vertically mounted array of the sensors could be used to obtain depth-dependent measures of the local population.

The bubble stream was generated by passing compressed air through a Hamilton 701RN laboratory syringe, which was additionally constricted at its base to provide the high-pressure drop necessary to give a repeatable size. ${ }^{16}$ This resulted in a bubble stream of resonant frequency $4300 \mathrm{~Hz}$, which was then allowed to rise through the transducer focus where it was insonified at a pump signal amplitude of 200 $\mathrm{Pa}$. The power spectral density of the signal scattered by the bubbles was then calculated, and the total energy contribution for each peak was estimated. The heights of the heterodyned imaging peak and the $\omega_{i}+\omega_{p}$ signals were measured over ten different sweeps and averaged, and were found to be repeatable to within $\pm 3 \%$ and $\pm 5 \%$, respectively.

Typical results for the laboratory measurements are shown in Fig. 3(a) and (b). Here the bubble stream was insonified between 3800 - and $4800 \mathrm{~Hz}$ pump frequency in 25 $\mathrm{Hz}$ steps, such that the bubbles passed through the transducer focus at a depth of $29 \mathrm{~cm}$. Figure 3(a) shows a mesh of the matrix formed by stacking the frequency responses of the heterodyned returned signals from adjacent pump frequencies together, and the locations of the $\omega_{i}, \omega_{i}+\omega_{p}$, and $\omega_{i}-\omega_{p}$ signals are labeled. Clearly evident is the heterodyned Doppler shifted imaging signal which plots as a continuous ridge to the left of the plot; this is constant over the 41 different pump frequencies. To the right of this are two broken ridges which rise up to a maximum value at $4300 \mathrm{~Hz}$ and then fall off again. These are the two combination frequency signals at $\omega_{i}+\omega_{p}$ and $\omega_{i}-\omega_{p}$. Peaks separation is
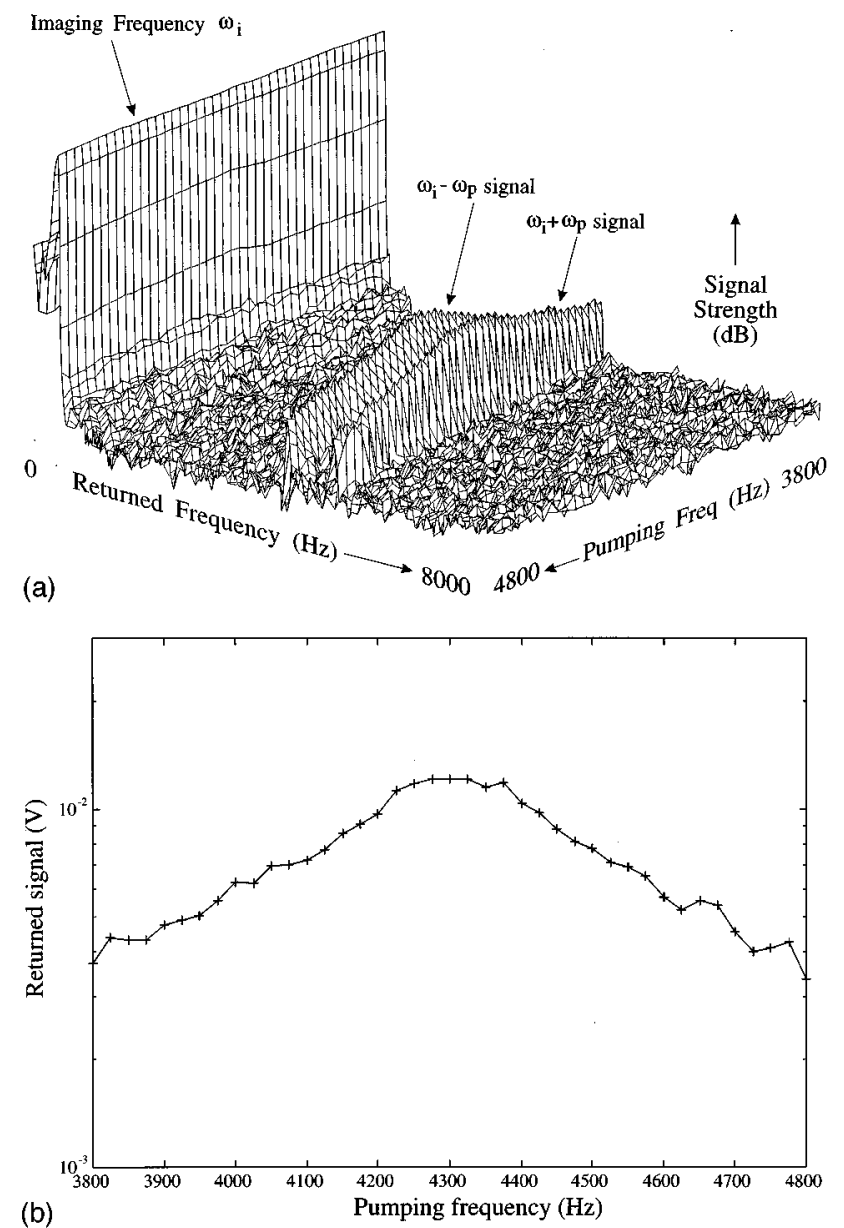

FIG. 3. (a) Typical results from the laboratory measurements on a rising bubble stream, with all the bubbles resonant at $4300 \mathrm{~Hz}$. The bubbles were insonified at 200-Pa amplitude between 3800 and $4800 \mathrm{~Hz}$ in $25-\mathrm{Hz}$ steps. (b) Height of the $\omega_{i}+\omega_{p}$ signal taken from the data in (a).

due to the Doppler shift on the scattered signal, which when heterodyned with the original imaging signal causes the $500-\mathrm{Hz}$ frequency offset. The amplitude of the $\omega_{i}+\omega_{p}$ signal is also shown in Fig. 3(b) over the pumping frequency range considered. These plots demonstrate an important property of the $\omega_{i} \pm \omega_{p}$ signal, the substantial off-resonant behavior of the signal. In order to calculate the number of bubbles at a specific radius per micrometer radial increment, which is the historical method of representing the data, this off-resonance effect must be removed. This is achieved by calculating the heights of the response of the bubbles whose radii lie on either side of the resonant bubble radius, and looking for the width of the radius spread before the signal strength falls off by $3 \mathrm{~dB}$. The effect of off-resonant bubbles contributing to the output signal can then be removed by dividing the measured bubble sound pressure levels by the 3-dB widths corresponding to that particular bubble radius.

The second stage of the calibration involved modeling the bubble-mediated sound pressure at the receiver transducer due to the two insonifying sound fields. The same bubble size and insonification conditions as employed in the laboratory experiments were used, such that a comparison of the results of the simulation with the experimentally estimated values would allow the validity of the model to be 


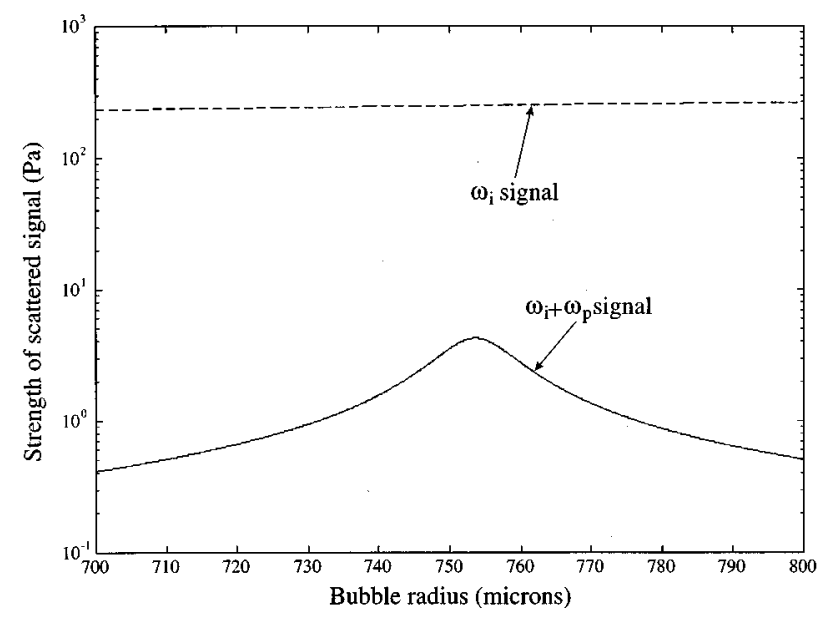

FIG. 4. Theoretically estimated amplitudes of the imaging signal and the $\omega_{i}+\omega_{p}$ signal for an insonifying sound field of frequency $4300 \mathrm{~Hz}$ and amplitude $200 \mathrm{~Pa}$, over a radius range of $700-800 \mu \mathrm{m}$.

examined and the sensitivity of the receiver transducer to be evaluated. The results from estimating the height of the $\omega_{i}+\omega_{p}$ and the imaging signal using the extended Rayleigh Plesset model are shown in Fig. 4. This is done for a range of bubble sizes from 700 to $800 \mu \mathrm{m}$ insonified by a $200 \mathrm{~Pa}$ amplitude sine wave of frequency $4300 \mathrm{~Hz}$. It is clear from the figure that the strength of the backscattered $\omega_{i}+\omega_{p}$ signal reaches a maximum of $4.3 \mathrm{~Pa}$ at a bubble radius of 754 $\mu \mathrm{m}$, and at this point the height of the scattered imaging signal is $251 \mathrm{~Pa}$. This can now be compared with the measurement of the scattered signal from the $4300-\mathrm{Hz}$ bubble stream, which showed an average height of the imaging signal of $1.27 \pm 0.04 \mathrm{~V}$ and an $\omega_{i}+\omega_{p}$ signal height of 17.3 $\pm 0.9 \mathrm{mV}$. Comparison of the heights of the two imaging signal strengths, and consideration of the frequency responses of the preamplifier and heterodyner, allow the sensitivity of the high-frequency transducer to be estimated at $13.4 \mu \mathrm{V} / \mathrm{Pa}$. As a method of testing the validity of the model, the difference in the strengths of the imaging signal and $\omega_{i}+\omega_{p}$ were also calculated. The ratio of the two signal components is $35.3 \mathrm{~dB}$ in the theoretical predictions, compared with $37.3 \mathrm{~dB}$ in the laboratory measurements, a 2-dB discrepancy which is equivalent to a $20 \%$ error in the $\omega_{i}+\omega_{p}$ pressure. If the damping was taken to comprise viscous losses alone, the ratio of the two signal heights would be less than $1 \mathrm{~dB}$, which is equivalent to a discrepancy of $>36 \mathrm{~dB}$. The contribution of the $2-\mathrm{dB}$ error to the final bubble number estimates will be discussed later.

Having verified the performance of the pulsation model, the sensitivity of the high-frequency receiver transducer can be estimated. Following this, the behavior of resonant bubbles at the four pump frequencies used in the oceanic tests of 28,50,60, and $88 \mathrm{kHz}$ were modeled (using parameters applicable to sea water rather than those of fresh water used in modeling the lab tests). With application of the same sensitivity adjustment and the relevant preamplifier/ heterodyner corrections, this provided an estimate of the signal levels expected from the different bubbles resonant at the four frequencies. This estimate relies on the sensitivity of the

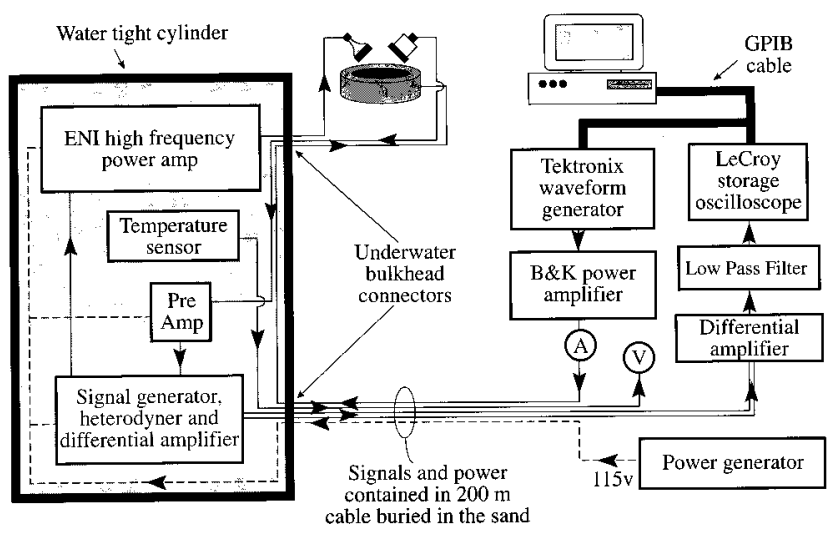

FIG. 5. Schematic of the apparatus used in the oceanic measurements.

receiver transducer being flat over the frequency range 1-1.1 $\mathrm{MHz}$ (as verified by the manufacturers data), and on the theory maintaining its validity over the greatly extrapolated frequency range considered.

\section{B. Oceanic data collection}

The equipment used in the sea trials was largely similar to that used in the laboratory experiments, and the schematic is shown in Fig. 5. The most important difference in the layout of the oceanic equipment is the provision of a remote equipment canister, which was set up in the sea and attached to the land-based equipment via an underwater bulkhead connector and $200 \mathrm{~m}$ of waterproof cable, as manufactured by PDM Group. The canister comprised a 1000-mm-long $\times 355$-mm-diam watertight aluminum alloy cylinder, which was painted to minimize corrosion, and clamped to a rigid scaffold structure as shown in Fig. 6. This canister contained the high-frequency power amplifier, the crystal oscillator and heterodyner equipment, the returned signal rf preamplifier, and a temperature sensor to monitor the effects that the enclosed space had on the potential of the equipment to overheat. Additionally, a differential amplifier pair was added to the returned signal circuit to ensure that no signal corruption occurred when passed down the 200-m cable: this additional step was analyzed in the laboratory and its frequency response quantified. Because of the higher-pump frequencies involved, the data were sampled at $500 \mathrm{kHz}$, and 50000

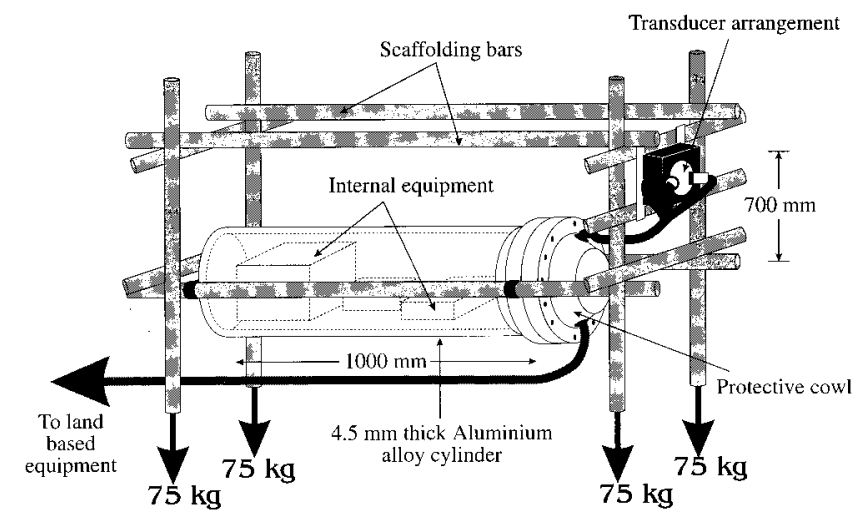

FIG. 6. Deployment details of the watertight canister and scaffolding. 
points were taken. To speed up the data collection and storage, the Tektronix output waveform comprised all four frequencies in one signal, and the LeCroy oscilloscope sampling the data was triggered by markers from the signal generator to allow the individual sections to be identified in the returned waveform.

Preliminary calibration tests to prepare the apparatus were carried out in an underground experimental tank, which measures $8 \mathrm{~m} \times 8 \mathrm{~m} \times 5 \mathrm{~m}$ deep. The equipment was lowered into the tank such that the transducer focus was $1.5 \mathrm{~m}$ underwater, the same depth as anticipated in the sea trials, and the pump signal amplitude measured with a constant input signal level at each of the four frequencies using a Bruel \& Kjaer 8103 hydrophone conditioned with a 2635 charge amplifier. This allowed the frequency response of the pump transducer to be inverted, and a constant and known pump signal amplitude to be employed.

The oceanic tests were performed in the North Sea between the 26th and 30th of November 1995, on a beach in Tunstall, East Yorkshire, and were carried out in tandem with a group from the Southampton Oceanography Centre. The beach was chosen due to its slight gradient, which allowed the equipment to be set up at low tide and anchored to the beach, such that as the tide came in it would eventually cover the rig to enable measurements to be taken. The rig was weighed down with $75 \mathrm{-kg}$ metal weights at each corner which were buried in the sand. The data collection used a 3000-Pa pump signal amplitude, and 25 four-frequency samples were taken over a 3.5 -min period every half hour while the transducers were immersed. As the signals were broadcast consecutively with no gap, each measurement lasted only $0.4 \mathrm{~s}$.

Every attempt was made to ensure that the scaffolding and canister created as little disturbance to the flow of water and bubble generation mechanisms as possible, by setting the transducers remote from the body of the canister and angling the ring transducer to the expected direction of the flow. The results presented in this text were taken at high tide when the transducers were $1.5-\mathrm{m}$ underwater, so even though the equipment setup had to be of robust construction due to the inhospitable nature of the local sea dynamics, the measurements should represent a reasonable sample of the bubble population there.

\section{RESULTS AND DISCUSSION}

A typical spectrum from the sea trials is presented in Fig. 7, taken from a $28-\mathrm{kHz}$ insonification. The particular data were collected at high tide (22.30) on the 29th of November 1995, when the wind speed at $10 \mathrm{~m}$ above sea level was $11 \mathrm{~m} / \mathrm{s}$, and the transducers were immersed at a depth of approximately $1.5 \mathrm{~m}$ in water approximately $3-\mathrm{m}$ deep. The data show the heterodyned signal from the high-frequency receiver, in which the imaging signal is visible at $1.5 \mathrm{kHz}$ (not at dc due to the Doppler shift from the moving bubble targets). The sum frequency spectral information contained just above the imaging signal is also shown, at approximately $29.5 \mathrm{kHz}$, and the difference data shown at $26.5 \mathrm{kHz}$. These signals would overlap were the measured bubbles stationary. Between the two combination frequency peaks is a

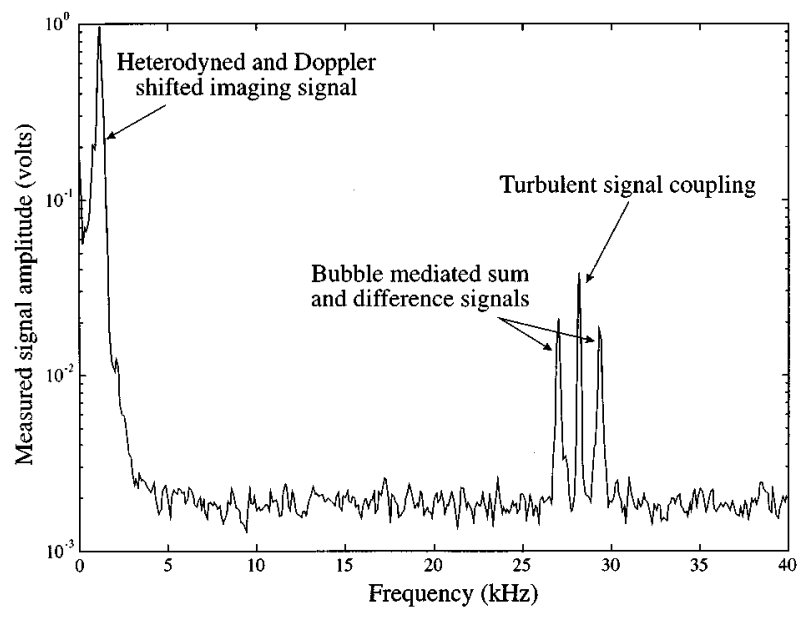

FIG. 7. Typical results from the oceanic measurements, showing the heterodyned frequency content. The particular data were collected at $28-\mathrm{kHz}$ pump frequency and an amplitude of $3000 \mathrm{~Pa}$. Clearly visible are the Doppler shifted imaging signal, the two combination frequency bubble indicators, and a single peak caused by turbulent coupling which is easily differentiated from the bubble-mediated peaks.

single spike at $28 \mathrm{kHz}$. This is caused by the nonlinear combination of the pump and imaging signals by turbulence in the detection zone, and can be therefore distinguished from the actual bubble-mediated information.

The data collected in each of the 25 time intervals were broken into 10 adjacent sections and the averaged power spectral density determined. These were then analyzed individually to get the energy contained in each of the heterodyned sum signals, and the result converted back to volts. Following that, the 25 samples were averaged to allow comparisons with existing time-averaged bubble data. This averaged measured voltage level was then corrected with the estimate for the sensitivity of the receiver transducer and the measured frequency responses of the preamplifier and heterodyner to give a measured bubble sound pressure level. This was converted into the number of bubbles per micrometer radius range by dividing the estimate with the theoretical $3-\mathrm{dB}$ spread of the $\omega_{i}+\omega_{p}$ signal, and scaled to give the number per unit volume by dividing by the estimated insonification volume. This was repeated at each of the four frequencies.

The data are shown in Fig. 8 compared with historical data, taken from Farmer and Vagle, ${ }^{5}$ Breitz and Medwin, ${ }^{17}$ and Johnson and Cooke ${ }^{18}$ who have all previously applied different bubble estimators to oceanic distributions. The plot shows the three sets of data superposed with the timeaveraged population measured using the two-frequency technique. The results show that the bubble population measured using the two-frequency technique considerably exceeds the other estimates over the whole radius range. This is to be expected as the data were collected in the surf zone where because of the continual wave action a high concentration of bubbles is created. Farmer and Vagle collected their data from bubble scatter in a 4-km-deep channel using upwards facing sonar designed to listen for the linear backscatter from the bubble population. The data presented were taken in a $12-14 \mathrm{~m} / \mathrm{s}$ wind speed. Johnson and Cooke used photo- 


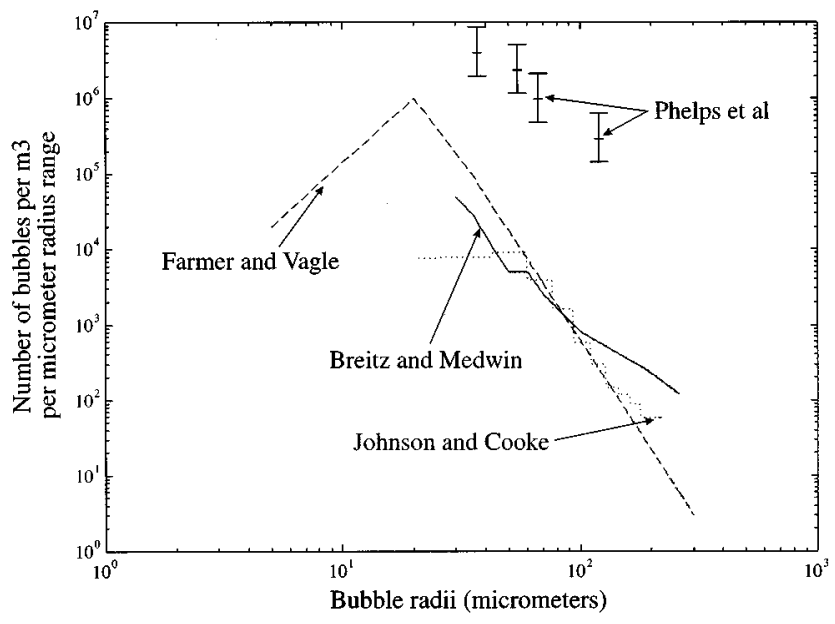

FIG. 8. Comparison of time-averaged data measured in the oceanic surf zone with historical estimates, taken from Farmer and Vagle, ${ }^{5}$ Breitz and Medwin, ${ }^{17}$ and Johnson and Cooke. ${ }^{18}$ The error bars on the surf zone measurements mostly reflect an uncertainty in the measurement volume. The bubble population is expressed as the number of bubbles of a specific radius over a $1-\mu \mathrm{m}$ range, per unit volume.

graphic estimates in 20-30-m-deep water, of which the population estimate at $0.7-\mathrm{m}$ depth and $11-13 \mathrm{~m} / \mathrm{s}$ wind speed is included. Breitz and Medwin collected their oceanic data with a flat plate resonator, which again exploits the linear resonance of bubbles. They measured in water $120-\mathrm{m}$ deep in $12-15 \mathrm{~m} / \mathrm{s}$ wind speeds and at a depth of $25 \mathrm{~cm}$. Thus, although the environmental measurement conditions were similar over the four sets of collected data, the local sea dynamics were very different for the Tunstall measurements owing to the presence of surging breakers. The heights of the error bars on the data points will be discussed in the next section.

A further advantage of the method employed here is the speed of the data collection. The data are collected in a series of 0.4-s "snapshots," being the time required to output the pump signal at all four frequencies and collect the heterodyned data onto the storage oscilloscope. This therefore allows the time variant nature of the bubble population to be investigated, rather than the technique requiring the time averaging inherent in earlier studies. Although the actual data acquisition is very fast, there is a longer delay caused by the transfer of the oscilloscope files onto the PC, of around $4 \mathrm{~s}$. For the data collected in the oceanic tests an additional 5-s delay was added between successive snapshots to enable a longer and therefore more representative section of time to be taken (of around $3 \frac{1}{2} \mathrm{~min}$ ), as this study was primarily concerned with measuring time-averaged data for comparison with earlier oceanic measurements. All the processing of the raw data was performed later. The time variant nature of the measurements is demonstrated in Fig. 9. The four different frequencies all show peaks in the signal at around $40 \mathrm{~s}$ and again at $140 \mathrm{~s}$, and all demonstrate a dip in the signal at $110 \mathrm{~s}$ and again at $160 \mathrm{~s}$. It should be noted that on occasions during the analysis the signal height peaked below the noise floor, and in these cases the particular readings have been left as gaps.

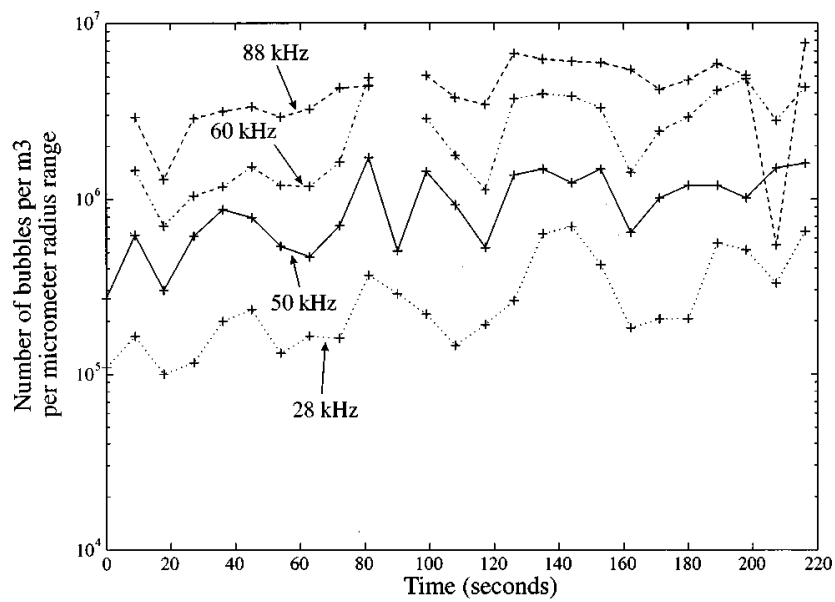

FIG. 9. Time-variant information at the four frequencies used, which represents resonant bubbles of radii $120,66,55$, and $37 \mu \mathrm{m}$. This is the same data as were averaged to produce Fig. 8, and therefore have the same error margins as the data on that plot.

\section{POSSIBLE SOURCES OF ERROR}

The data collection technique was employed to measure a real bubble population in the sea, and as a preliminary data collection exercise the bubble measurement apparatus was incorporated into a surf zone monitoring program which was being conducted by the Southampton Oceanographic Centre. Thus the field data were necessarily collected in a dense bubble population. To prevent the bubble signals being screened by nearby bubbles in this dense environment, the high-frequency transducers had to be located close to the intended measurement area, and eventually a distance of 90 $\mathrm{mm}$ from the faces of both the transmitter and receiver transducers was chosen. However, as the high-frequency crystals are 31- and 28-mm-diam, respectively, this measurement volume is in the near field of both. This represents a trade-off in the measurement procedure, as although the bubble sizer gave the required high-spatial resolution and signal strength, the actual determination of the insonification volume is considerably harder to estimate, and the experimental insonification conditions are less well-defined. This gives an error associated with the data collection which is larger than that which would be achieved in more typical measurement conditions, where the risk of signal corruption and screening is less, allowing the transducers to be set to insonify a volume of fluid which is in the far field of each. The analytical estimate of the insonification volume of $0.2 \mathrm{~cm}^{3}$ is taken to be where the combined sensitivity of the two transducers falls to $3 \mathrm{~dB}$ of the maximum level, and it is considered that the volume estimate is only accurate to $+200 \%$ and $-50 \%$, which is reflected in the height of the error-bars in Fig. 8.

A further systematic error is included in the calibration of the hydrophone sensitivity using the comparison of the theoretical model and the laboratory measured data. The predicted height of the $\omega_{i}+\omega_{p}$ signal is $20 \%$ higher than the measured data, suggesting either that even with the revised model the damping is still not perfectly represented or that the laboratory insonification conditions were imperfectly known. Harder to evaluate is the effect of this discrepancy as 
the bubble sizes get smaller. For the high-resonance frequency bubbles, the primary source of the damping is thermal losses, which is the least well-defined of the three mechanisms. As an indication, the assumed value for $\kappa$ for the $88-\mathrm{kHz}$ bubbles (estimated using Eller's theory) is 1.23, but using the analysis presented by Prosperetti ${ }^{19}$ in his reworking of the thermal effects in a forced bubble, it is 1.25. It would appear that at the low driving amplitudes employed, this is not sufficiently different to contribute an error of the same order as the volume estimate, although the systematic error of $20 \%$ has been included in the error spread calculations over the entire frequency range.

Also as the bubble radii become smaller, the effects of surface tension become much larger, and the model becomes more dependant on a less clearly defined parameter. Thorpe $^{20}$ supposes that over the course of a few tens of seconds "clean" bubbles of small radius in the sea become "dirty," that is coated in surfactant, and gives an estimate for the surface tension parameter $\sigma$ of $0.036 \mathrm{~N} / \mathrm{m}$ for these dirty bubbles, and $0.072 \mathrm{~N} / \mathrm{m}$ for the clean bubbles. He estimates that the lifetimes of bubbles are considerably longer than the time it takes to become dirty, and therefore treats all bubbles as such, but it is evident that under a breaking wave such as experienced in these experimental conditions, the surfactant coating of the measured bubbles is harder to determine. However, this will not contribute significantly to the measurement error, as the smallest bubble size considered in the tests is still large enough not to be affected overly by this. For example, for those bubbles resonant at $88 \mathrm{kHz}$, the Laplace pressure inside a motionless clean bubble due to the surface tension is $\sim 4000 \mathrm{~Pa}$, i.e., approximately $4 \%$ of the total internal pressure. As the bubbles in the ocean will fall between the two surface conditions, the surface tension should only account for between $2 \%$ and $4 \%$ of the internal pressure even for these smallest bubbles.

Though measured in our experiment, it is apparent that certain insonification parameters need not be well-defined, as the same transducer arrangement is used in both the laboratory calibration and the actual sea trials. An example is the absolute amplitude of the imaging signal at $30 \mathrm{kPa}$, as the theory indicates that the height of the $\omega_{i}+\omega_{p}$ signal scales linearly with this parameter. Thus an error in the estimate of the amplitude will affect the calculated sensitivity of the high-frequency transducer, but this effect will be removed when the sensitivity correction is applied to the oceanic bubbles. Another parameter which will be insensitive to poor definition in the model is the distance of the bubbles from the measurement transducer, although this will affect the calculation of the insonification volume.

\section{CONCLUSIONS}

The paper has described the design and implementation of an oceanic bubble measurement device capable of sampling a small volume, and presented the results taken from initial studies in the shallow and high bubble density surf zone. Unlike previous oceanic measurements, the technique employed here provides an unambiguous and potentially more accurate estimate of the local bubble population. Additionally, as the device collects data over a period of only 0.4 $\mathrm{s}$ for all four frequencies, the results can be used to investigate the temporal changes in the bubble distribution. To our knowledge, these are the first measurements of the bubble spectral density in the shallow surf zone, and it is planned to mount the equipment from a buoy in the deeper ocean where historical studies have been made, which will enable comparative data to be collected. The main source of error in the estimates of the population arises through an imperfect knowledge of the insonification volume, as the high bubble densities measured required the employment of a very small insonification volume. This was necessarily located in the near field of both high-frequency transducers. However, this limitation will be removed for the planned lower density studies by the use of a larger measurement volume.

\section{ACKNOWLEDGMENTS}

The authors wish to extend their thanks to David Baldwin, Alan Hall, and Professor Steve Thorpe from the Southampton Oceanography Centre for their assistance with the beach tests, and to Rob Stansbridge, Daniel Russell, John Taylor, Mike Bartlett, Dave Edwards, John Hawkes, and the Southampton University Physics Technical Workshop for their help with the design and manufacture of the oceanic rig. Thanks also are extended to Dr. Ulrich Pollmann for helpful discussion. The work was funded using a NERC award (ref. GR3 09992), and Mr. Ramble was funded by EPSRC (ref. GR/H 79815).

${ }^{1}$ D. K. Woolf, "Bubbles and the air-sea transfer velocity of gases," Atmosphere-Ocean 31, 451-474 (1993).

${ }^{2}$ T. J. Lin and H. G. Donnelly, "Gas bubble entrainment by plunging laminar liquid jets," AIChE. J. 12, 563-571 (1966).

${ }^{3}$ E. O. Belcher, "Quantification of bubbles formed in animals and man during decompression,'” IEEE Trans. Biomed. Eng. 27, 330-338 (1980).

${ }^{4}$ T. G. Leighton, The Acoustic Bubble (Academic, London, 1994).

${ }^{5}$ D. M. Farmer and S. Vagle, "Waveguide propagation of ambient sound in the ocean-surface bubble layer," J. Acoust. Soc. Am. 86, 1897-1908 (1989).

${ }^{6}$ T. G. Leighton, D. G. Ramble, and A. D. Phelps, "The detection of tethered and rising bubbles using multiple acoustic techniques," J. Acoust. Soc. Am. (in press).

${ }^{7}$ A. D. Phelps and T. G. Leighton, "High resolution bubble sizing through detection of the subharmonic response with a two frequency excitation technique," J. Acoust. Soc. Am. 99, 1985-1992 (1996).

${ }^{8}$ V. L. Newhouse and P. M. Shankar, "Bubble sizing using the nonlinear mixing of two frequencies,'” J. Acoust. Soc. Am. 75, 1473-1477 (1984).

${ }^{9}$ J. Y. Chapelon, P. M. Shankar, and V. L. Newhouse, "Ultrasonic measurement of bubble cloud size profiles,' J. Acoust. Soc. Am. 78, 196-201 (1985).

${ }^{10}$ P. M. Shankar, J. Y. Chapelon, and V. L. Newhouse, "Fluid pressure measurement using bubbles insonified by two frequencies,' Ultrasonics 24, 333-336 (1986).

${ }^{11}$ D. Koller, Y. Li, P. M. Shankar, and V. L. Newhouse, "High speed bubble sizing using the double frequency technique for oceanographic applications," IEEE J. Oceanic Eng. 17, 288-291 (1992).

${ }^{12}$ B. E. Noltingk and E. A. Neppiras, "Cavitation produced by ultrasonics," Proc. Phys. Soc. London, Ser. B 63, 674-685 (1950).

${ }^{13}$ E. A. Zabolotskaya and S. I. Soluyan, "Emission of harmonic and combination-frequency waves by air bubbles," Sov. Phys. Acoust. 18, 396-398 (1973)

${ }^{14}$ E. A. Neppiras, “Acoustic cavitation,” Phys. Rep. 61, 159-251 (1980).

${ }^{15}$ A. I. Eller, "Damping constants of pulsating bubbles," J. Acoust. Soc. Am. 47, 1469-1470 (1970).

${ }^{16}$ R. Clift, J. R. Grace, and M. E. Weber, Bubbles, Drops and Particles (Academic, New York, 1978). 
${ }^{17}$ N. Breitz and H. Medwin, "Instrumentation for in situ acoustical measurements of bubble spectra under breaking waves,' J. Acoust. Soc. Am. 86, 739-743 (1989).

${ }^{18}$ B. D. Johnson and R. C. Cooke, "Bubble populations and spectra in coastal waters; a photographic approach,' J. Geophys. Res. 84, 37613766 (1979).
${ }^{19}$ A. Prosperetti, "Thermal effects and damping mechanisms in the forced radial oscillations of gas bubbles in liquids," J. Acoust. Soc. Am. 61, 17-27 (1977).

${ }^{20} \mathrm{~S}$. A. Thorpe, "On the clouds of bubbles formed by breaking wind-waves in deep water, and their role in air-sea gas transfer," Philos. Trans. R. Soc. London, Ser. A 304, 155-210 (1982). 